\title{
TWO CASES OF CONGENITAL ATRESIA OF THE FORAMINA OF MAGENDIE AND LUSCHKA
}

\author{
BY
}

\author{
A. F. J. MALONEY
}

From the Department of Neuropathology, University of Leeds

Obstruction of the foramina in the roof of the fourth ventricle is a well recognized cause of hydrocephalus and is usually due to inflammation of the meninges at the base of the brain. In cases of meningitis the foramina may be blocked in the acute stage by exudate, or, in later stages, if resolution is incomplete, by fibrous granulation tissue. In a few cases a history of preceding meningitis cannot be obtained, and at necropsy the meninges at the base of the brain show little or no evidence of preceding inflammation. The foramina cannot be demonstrated and the entire ventricular system is dilated and the lateral recesses ballooned. It has been suggested that such cases either result from a developmental anomaly at the hind end of the ventricle or that there has been a mild, low-grade meningitis unaccompanied by neurological symptoms. In only a few, where examination has included histological study, can the claim for a developmental abnormality be sustained.

Russell (1949) recorded the case of a baby girl aged 1 year in whom hydrocephalus resulted from the presence of a neuroglial septum, incompletely lined by ependyma, which occluded the hind end of the fourth ventricle. She also drew attention to Hilton's case, probably the earliest to be described (Hilton, 1887), in which the foramen of Magendie was occluded by a membrane.

Taggart and Walker (1942) described three cases in which they believed that the hydrocephalus was due to atresia of the foramina of Magendie and Luschka. The striking features of their cases were (1) cystic dilatation of the ventricle which almost filled the entire posterior fossa, (2) malformation of the vermis which was quite small-apparently representing only the anterior vermis-and was displaced upwards by and incorporated in the wall of the cystic ventricle, (3) the high position of the tentorium and lateral venous sinuses. No trace of the posterior vermis was seen nor were the foramina of Magendie and Luschka patent. These authors stated that this condition had been rarely described and apart from two doubtful cases at the end of the last century they were able to find reference to only six cases in more recent times, namely those of Dandy and Blackfan (1914), Pines and Surabaschwili (1932), Castrillón (1933), Scarff (1933), Sahs (1941), and Cohen (1942). Not all of the authors, however, were prepared to accept, or indeed even considered, the theory that both the anomaly of the vermis and the cystic dilatation of the ventricle were secondary to atresia of the foramina. Thus Pines and Surabaschwili (1932), Castrillón (1933), and Sahs (1941) all described their cases as primary aplasia of the cerebellar vermis.

The purpose of this paper is to describe two patients with this unusual condition, who were treated by Mr. W. R. Henderson in the Department of Neurosurgery, The General Infirmary at Leeds, one of whom is still alive, and to draw attention to five further cases described in the recent literature by Shryock and Alexander (1943, one case), Walker (1944, one case), Sidenberg, Kessler, and Wolpaw (1946, one case), and Coleman and Troland (1948, two cases).

\section{Case Reports}

Case 1.-The patient was a girl aged 6 years, who until 14 months before had been in good health and had developed normally. She then started to complain of vomiting, especially first thing in the morning. For five weeks before admission she had suffered from increasingly severe headaches. She was admitted to the Neurosurgical Unit on August 29, 1951.

The head was moderately enlarged; there was a cracked-pot note, bilateral papilloedema, and paresis of the right external rectus muscle. There was also slight ataxia of the left arm and the left leg.

The cerebrospinal fluid was normal.

Ventriculography.-The lateral and third ventricles were grossly enlarged and a large collection of air was seen in the posterior fossa (Fig. 1). The line of attachment of the tentorium to the side wall of the posterior fossa 


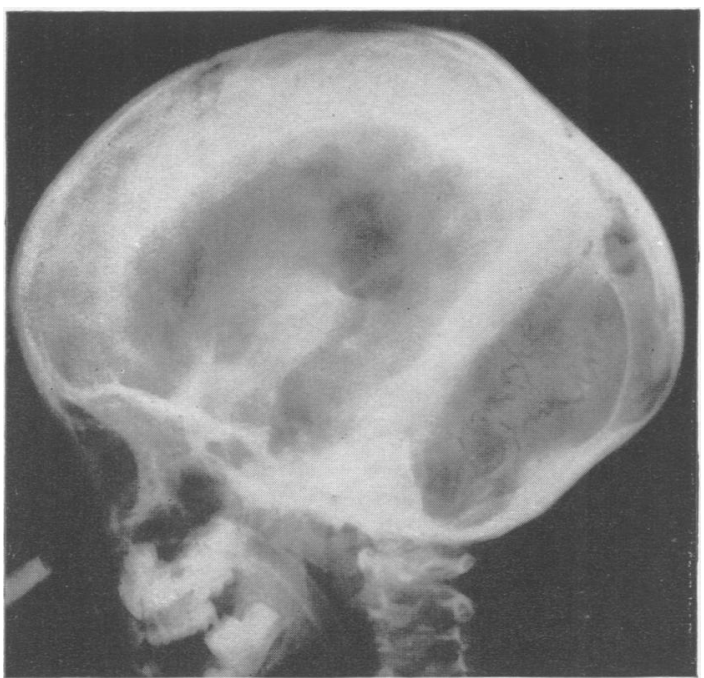

FIG. 1.-Case 1 : ventriculogram showing dilatation of lateral and third ventricles and a large collection of air in the posterior fossa. The tentorium is elevated.

appeared unusually elevated and, moreover, the initial burr-holes, made for ventriculography in what was thought to be the usual position, had exposed the lateral sinus on either side, indicating that the sinuses were higher than normal.

Operation.-The posterior fossa was explored. It was noted that the left side of the posterior fossa bulged rather more than the right and it was only when the bone was removed that it became apparent that the median structures were $2 \mathrm{~cm}$. to the right of the midline. Beneath the dura a large cyst was found lined by a delicate membrane resembling arachnoid. The cyst was in direct communication with and formed part of a greatly enlarged fourth ventricle. The cerebellar hemispheres were $2 \mathrm{~cm}$. apart and between them high up in front a flattened band of tissue, thought to be vermis, was seen. Nothing further was done.

The child died 12 hours after operation.

Necropsy.-The only abnormalities noted were in the head.

The asymmetry of the posterior fossa noted at operation and necropsy corresponded to the asymmetrical expansion of the fourth ventricle. The lateral sinuses and the attachment of the tentorium to the side wall of the posterior fossa were abnormally high.

The ventricle, which resembled a very large cyst, was enormously dilated. A large part of the cyst wall had been removed at operation and only small fragments remained along the line of its attachment to the dorsum of the cerebellar hemispheres, anterior vermis, and dorsal aspect of the upper end of the medulla (Fig. 2).

No trace of the posterior vermis was seen. The anterior vermis was rudimentary and had been displaced upwards and slightly to the right of the midline, so that it lay horizontally. It was difficult to identify the individual lobules, but the vermis probably comprised the lingula, central lobe, culmen, and part of the declive.

The cerebellum had been displaced slightly to the right, and the left hemisphere lay at a higher level than the right elevating the tentorium into the cleft between the ventromedial surfaces of the occipital lobes. The hemispheres were slightly smaller than normal and had been displaced laterally, upwards, and forwards by the expanded ventricle. The maximum diameter of each was $5 \mathrm{~cm}$. The pattern of the cerebellar folia appeared normal and the arachnoid was not thickened.

As a result of these abnormalities the whole of the expanded ventricle from obex below to aqueduct above was exposed to view. Its lateral walls were formed by the medial surfaces of the separated cerebellar hemispheres.

The cyst wall was quite thin and semi-transparent. In the midline rostrally it was attached to the posterior extremity of the rudimentary vermis; from here the line of reflection extended on either side obliquely in a lateral and posterior direction to reach the extreme lateral aspect of the cerebellar hemisphere near its caudal tip. On the left side it then passed round the lateral border of the hemisphere in an anterior and rostral direction closely following the line of the horizontal fissure till it reached the left lateral recess. Here the membrane had disappeared, but it was seen again attached to the margin of the left restiform body, passing dorsally and medially round the upper end of the medulla towards the obex. On the right side the line of attachment passed from the caudal tip of the cerebellar hemisphere inferiorly along the posterior margin of its medial surface, then anteriorly and rostrally along its inferior margin to the right lateral recess. It then ran posteriorly around the upper end of the medulla to become continuous with the line of attachment already followed to the obex on the left side.

At its line of attachment the membrane was composed

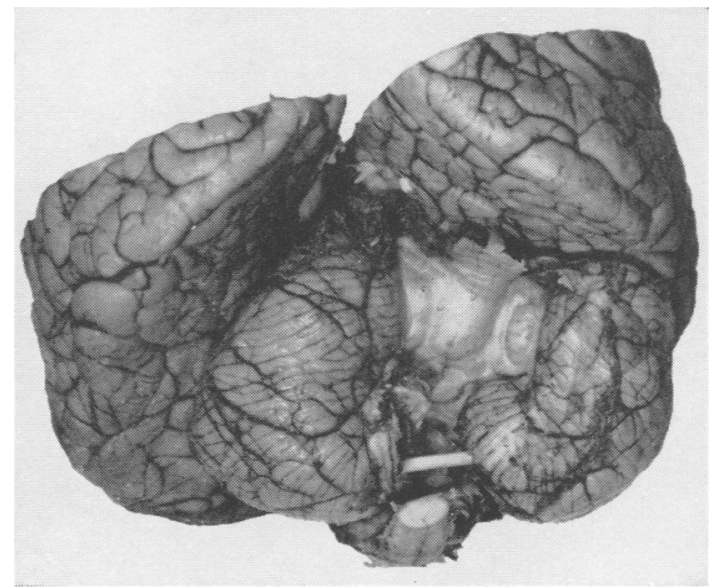

FIG. 2.-Case 1: cerebellum viewed from below and behind. The cyst wall has been almost entirely removed to expose the dilated fourth ventricle and the rudimentary anterior vermis displaced upwards and to the right. 
of two layers, an inner reflected rostrally on to the under surface of the vermis, caudally on to the floor of the ventricle, and laterally on to the medial surface of the cerebellar hemispheres, eventually becoming continuous with the ependyma lining the ventricle itself. The outer layer was reflected rostrally on to the upper surface of the vermis, caudally on to the medulla and laterally on to the lateral surface of the cerebellar hemispheres. These layers are too thin and transparent to be seen in the photograph (Fig. 2). Too much of the cyst wall had already been removed to enable the position of the foramina of Magendie and Luschka to be identified, and the cisterna magna was not seen.

The lateral and third ventricles were markedly dilated. The leptomeninges were healthy.

Microscopy.-The double membrane of the cyst wall (Fig. 3) is composed externally of a layer of vascular collagenous fibrous tissue and internally of a thin layer of neuroglial tissue incompletely lined on its inner aspect by a single row of ependymal cells, columnar in some places, flattened in others. At the site of attachment of the membrane to the cerebellar hemispheres, these two layers are reflected in opposite directions, the neuroglial layer passing medially towards the floor of the ventricle, the fibrous layer becoming continuous with the arachnoid on the lateral aspect of the hemispheres (Fig. 4). The pia formed no part of the cyst wall.

Case 2.-The patient was a girl aged 13 years who was well until three weeks before admission, when she began to complain of increasingly severe headache. later accompanied by vomiting and periodic drowsiness. She was admitted to the Neurosurgical Unit on November $12,1948$.

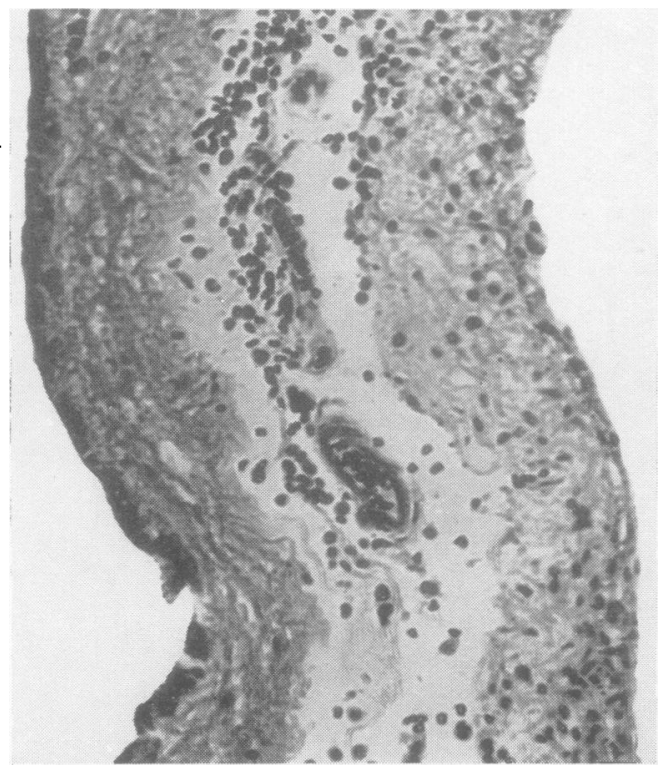

FiG. 3.-Case 1: section of cyst wall, showing neuroglial layer lined by ependyma on one side and vascular fibrous tissue on the other (hacmatoxylin and eosin. $\times 190$ ).
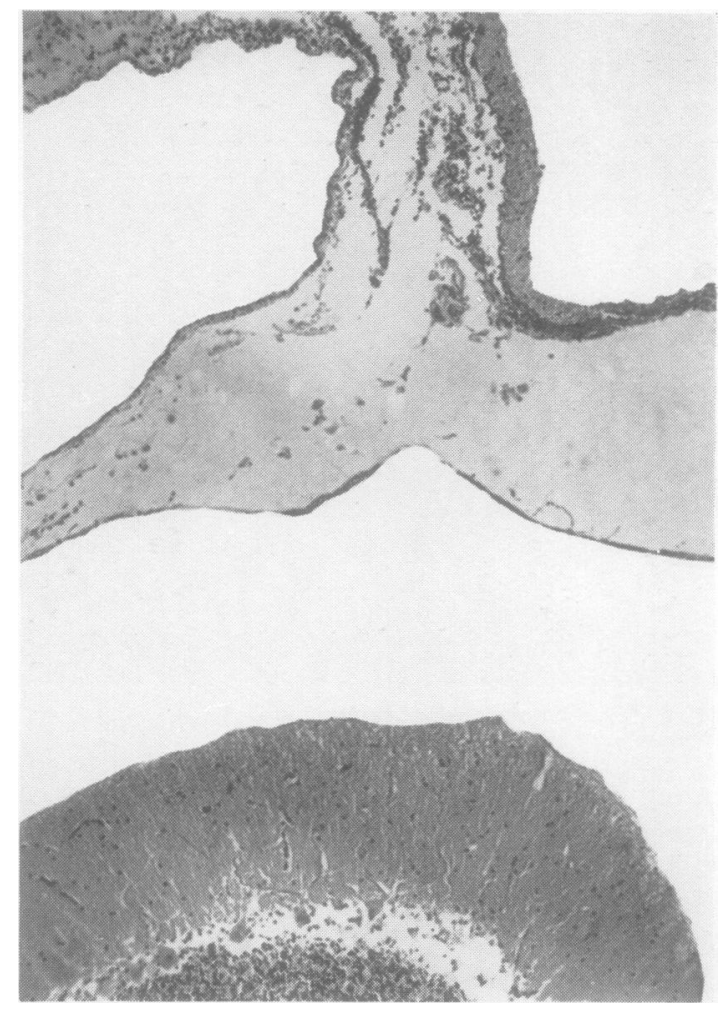

Fig. 4.-Case 1 : attachment of the double-layered cyst wall to the cerebellum. Note reflexion of the slightly thicker neural layer in one direction and the delicate arachnoidal layer in the other. (Haematoxylin and eosin. $\times 90$.)

There was bilateral papilloedema, bilateral complete sixth-nerve paralysis, and the right plantar reflex was extensor. There was no ataxia. The head was obviously slightly enlarged. The cerebrospinal fluid was normal.

Ventriculography.-The ventricles (Fig. 5) were dilated and there was a large collection of air in the posterior fossa outlining the cerebellar hemispheres below and what appeared to be vermis above. The burr-holes were almost directly over the lateral sinuses, indicating that the latter and the tentorial attachment were in an unusually high position.

Operation.-The posterior fossa was explored and a large, cyst-like structure containing clear fluid and air was encountered beneath the dura. It was formed by a delicate membrane bridging the gap between the cerebellar hemispheres and was apparently an expanded fourth ventricle. When the membrane was incised the cerebellar hemispheres were easily displaced laterally and the whole of the ventricle was brought into view because the vermis was deficient and was compressed upwards and forwards almost to the tentorial opening.

A portion of the cyst was removed and a small window cut in the tentorium. 


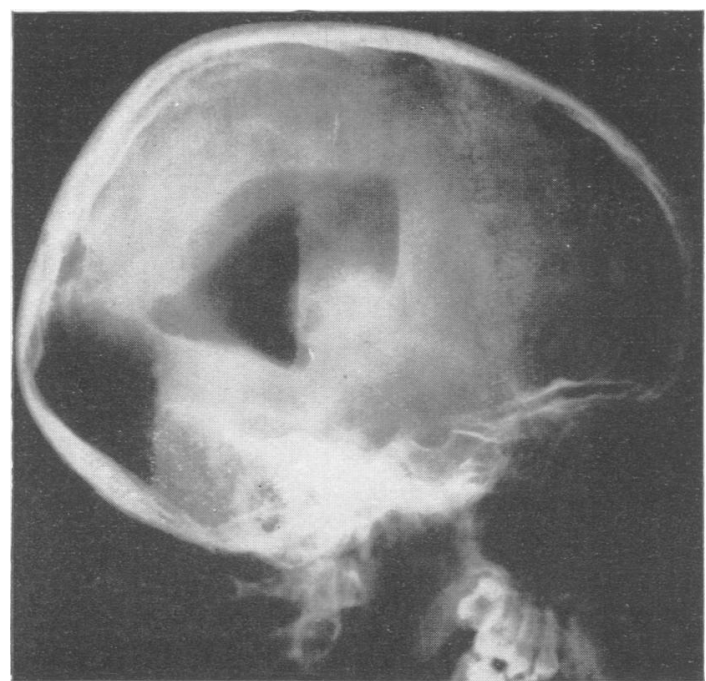

Fig. 5.-Case 2: ventriculogram showing hydrocephalus and a large collection of air in the posterior fossa. The tentorium is raised.

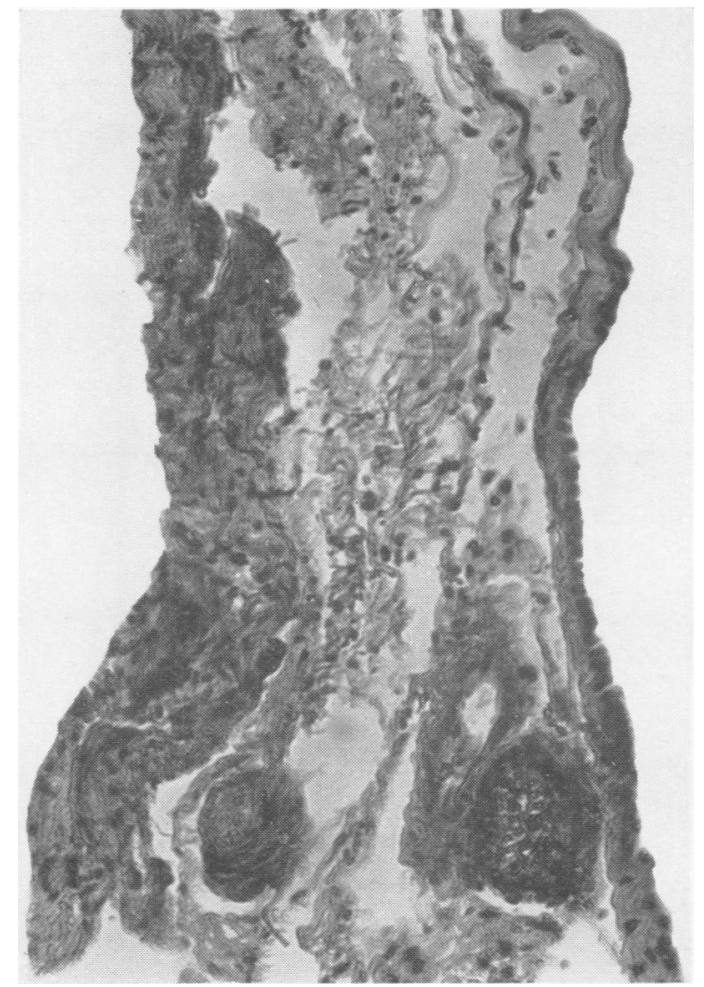

FIG. 6.-Case 2 : portion of the cyst wall removed at operation. Note distinct collagenous and neuroglial layers; the latter is lined by ependymal cells. (Haematoxylin and eosin. $\times 190$.)
The child made an uninterrupted recovery and is alive and well four years later. There are no abnormal neurological signs.

Microscopy.-The cyst wall (Fig. 6) removed at operation was similar in appearance to that found in the first case, being composed of a double layer of collagenous and neuroglial tissue.

The second case is apparently similar to the first. Both children presented with signs of raised intracranial pressure, namely, headache, vomiting, and papilloedema without localizing signs, although in Case 1 there was slight ataxia of the left arm and leg. In Case 1 the symptoms were progressive over a period of 14 months, in Case 2 they were present for only three weeks. In both cases the previous medical history was uneventful. Birth and development were normal and there was no history of meningitis. The head of each child was slightly enlarged, but this appears to have escaped notice until admission to hospital. A pre-operative diagnosis was not made in either case, but in retrospect the appearances on ventriculography are believed to be characteristic of this condition in its fully established form, namely, (1) dilatation of the entire ventricular system, (2) a large collection of air in the posterior fossa, (3) a high position of the tentorium and lateral sinuses, which may be observed when the burr-holes are made. Similar $x$-ray observations were made by Taggart and Walker (1942). At operation a large, thinwalled, membranous cyst, filling the posterior fossa, and a rudimentary vermis were found in each case.

\section{Discussion}

The cyst may at first be mistaken for a distended cisterna magna, but this idea is readily corrected by further examination, for not only is the cyst found to form part of a dilated ventricle, but histologically it is lined by neuroglia and ependyma on its inner surface. Moreover, in a few cases a normal cisterna magna has been identified below the cyst (Dandy, 1921; Taggart and Walker, 1942).

When the cyst has been correctly recognized as a dilated ventricle, some authors (Pines and Surabaschwili, 1932; Castrillón, 1933; Sahs, 1941, Sidenberg and others, 1946) have considered that the primary abnormality is congenital aplasia of the cerebellar vermis and that the cystic dilatation of the ventricle is secondary, while others (Dandy and Blackfan, 1914; Taggart and Walker, 1942 ; Walker, 1944; Coleman, and Troland, 1948) have recognized that the enlargement of the ventricle is dependent upon occlusion of the foramina in its roof, and that the anomaly of the vermis is secondary.

The aplasia of the vermis indicates that the condition must arise early in intra-uterine life. If 
the primary anomaly affected the vermis it would be difficult to explain the cystic dilatation of the ventricle and the occlusion of the foramina, which do not occur with primary aplasia of the cerebellar vermis. The alternative view that absence or delayed opening or early occlusion in utero will be followed not only by hydrocephalus but by failure of the vermis to develop in a normal manner is supported by embryological concepts. This line of reasoning has been adequately dealt with in the paper by Taggart and Walker (1942), in which reference will be found to Weed's experiments on the development of the fourth ventricle and subarachnoid space in the pig, and to the observations of others in the human embryo.

Enlargement of the contents of the posterior fossa in utero may also produce secondary changes in the skull and position of the transverse sinuses which are of diagnostic importance to the surgeon. In the normal developing embryo there is a gradual posterior migration of the transverse venous sinuses to their adult position on the occipital bone and postero-inferior angle of the parietal bone which is attained at birth (Streeter, 1915). Dilatation of the fourth ventricle at an early stage may prevent this and the sinuses may remain higher up on the inferior part of the parietal bone where their imprint can sometimes be seen on radiography.

It might be expected that this condition would cause manifest symptoms in infancy and death in early life, but study of the reported cases shows that this is not so. The age at death has ranged from a few months (Russell, 1949 ; Dandy and Blackfan, 1914) to almost 60 years (Castrillón, 1933) though the majority have been observed during the first two decades. Some have apparently developed normally or had only slight periodical symptoms of raised intracranial pressure, sometimes associated with an enlarging head, before the onset of the final illness, while others have obviously been abnormal from or soon after birth. Why the onset of symptoms should be delayed is not immediately apparent, but the following explanations have been offered :(a) The cyst may act as a semipermeable membrane and allow the passage of some cerebrospinal fluid from the ventricle into the subarachnoid space. (b) It has been observed in some cases that the choroid plexus of the fourth ventricle is unusually small and therefore the quantity of cerebrospinal fluid produced may be less. (c) The atresia may not be absolute and some cerebrospinal fluid may escape through greatly narrowed foramina.

There is nothing in the clinical history of these patients to suggest the nature of the underlying condition responsible for the hydrocephalus. If the abnormally high position of the transverse sinuses is recognized radiographically, then the condition should be suspected. In the typical case the ventriculographic appearances are characteristic. It is of interest to note, that in neither of the cases described by Coleman and Troland (1948) were these radiological appearances present, and the authors suggested that this was probably due to early equilibrium allowing normal migration of the sinuses.

The operative mortality is unfortunately high, though the technical procedure of removing part of the cyst membrane and allowing free communication between the fourth ventricle and the subarachnoid space is relatively simple and has proved effective in some cases. Successful results have been recorded by Scarff (1933), Cohen (1942), Walker (1944), and Coleman and Troland (1948) (two cases), in addition to the second case reported here.

\section{Summary}

Two cases are described of an anomaly of the cerebellar vermis associated with cystic dilatation of the fourth ventricle and hydrocephalus.

Reference is made to similar cases reported in the literature and reasons are given for the belief that the condition is due to partial or complete failure of the foramina of Magendie and Luschka to become patent at or about the fourth month of intra-uterine life.

The clinical and radiographic features are discussed. The appearances on ventriculography are considered to be diagnostic of the condition.

One patient is alive and well four years after operation, the other died shortly afterwards.

I wish to thank Mr. W. R. Henderson and Professor R. A. Willis for their encouragement and advice during the preparation of this paper, Mr. W. H. Lawson for technical assistance, and the Department of Medical Photography, University of Leeds, for the photographs. Case 1 was referred to the Department of Neurosurgery by Dr. E. C. Allibone and Case 2 by Dr. J. A. Price.

\section{REFERENCES}

Castrillón H., A. (1933). Z. ges. Neurol. Psychiat., 144, 113.

Cohen, I. (1942). J. Mt Sinai Hosp., 8, 441.

Coleman, C. C., and Troland, C. E. (1948). J. Neurosurg., 5, 84.

Dandy, W. E. (1921). Surg. Gynec. Obstet., 32, 112.

, and Blackfan, K. D. (1914). Amer. J. Dis. Child., 8, 406.

Hilton, J. (1887). Rest and Pain-A Course of Lectures, 4 th ed., p. 38 Edited by W. H. A. Jacobson. Bell, London.

Pines, L., and Surabaschwili, A. (1932). Arch. Psychiat. Nervenkr., 96, 718.

Russell, D. S. (1949). Spec. Rep. Ser. med. Res. Coun. Lond., No. 265. p. 19 .

Sahs, A. L. (1941). Arch. Path. Lab. Med., 32, 52.

Scarff, J. E. (1933). J. nerv. ment. Dis., 78, 400.

Shryock, E. H.. and Alexander, H. B. (1943). Bull. Los Angeles neurol.

Soc., 8, 11 . 28, 719 .

Streeter, G. L. (1915). Amer. J. Anat. 18, 145.

Taggart, J. K., and Walker, A. E. (1942). Arch. Neurol. Psychiat., Chicago, 48, 583.

Walker, A. E. (1944). J. Neuropath., 3, 368. 